\title{
Clinical and Economic Consequences of Inhaled Corticosteroid Doses and Particle Size in Triple Inhalation Therapy for COPD: Real-Life Study
}

This article was published in the following Dove Press journal: International Journal of Chronic Obstructive Pulmonary Disease

\author{
Antoni Sicras-Mainar (D) \\ Francisco J de Abajo (iD) 2,3 \\ José Luis Izquierdo- \\ Alonso id ${ }^{4,5}$ \\ 'Health Economics \& Outcomes \\ Research, Atrys Health, Barcelona, Spain; \\ ${ }^{2}$ Unidad de Farmacología Clínica, \\ Hospital Universitario Príncipe de \\ Asturias, Madrid, Spain; ${ }^{3}$ Departamento \\ de Ciencias Biomédicas, Universidad de \\ Alcalá (IRYCIS), Madrid, Spain; \\ ${ }^{4}$ Departamento de Medicina \\ y Especialidades, Universidad de Alcalá, \\ Madrid, Spain; ${ }^{5}$ Servicio de Neumología, \\ Hospital Universitario de Guadalajara, \\ Guadalajara, Spain
}

Objective: To determine the clinical and economic consequences of inhaled corticosteroid doses and particle size in patients on triple-inhalation therapy for COPD.

Methods: Patients aged $\geq 40$ years who initiated treatment with multi-inhaler triple-inhaled therapy between 1 January 2015 and 31 March were included and followed for 1 year. Patients were grouped according to inhaled corticosteroid (ICS) dose (low/medium/high) and particle size device (extrafine/non-extrafine particles). Outcome variables were moderate and severe exacerbations, pneumonia and healthcare resource use (HCRU) costs. A multivariate analysis was performed for model correction $(\mathrm{p}<0.05)$.

Results: A total of 2185 patients (mean age 72.3 years, $82.9 \%$ male) were analysed. Of these, 849 (38.9\%) patients received low-dose ICS, 612 medium-dose ICS (28.0\%) and 724 (33.1\%) high-dose ICS. Exacerbations occurred more frequently with increasing IC dose (low: $26.4 \%$, medium: $28.7 \%$ and high: $30.4 \%$; $\mathrm{p}=0.047$ ), as did the proportion of pneumonia $(3.4 \%, 4.2 \%$ and $6.9 \%$, respectively $(\mathrm{p}=0.041))$. The annual mean cost/unit was $€ 2383$ for low dose, $€ 2401$ for medium dose and $€ 2625$ for high dose $(\mathrm{p}=0.024)$. Four hundred and sixty-two (31.6\%) patients used an extrafine particle device and $999(68.4 \%)$ a non-extrafine particle device: the proportion of exacerbations was $24.0 \%$ vs $30.4 \%(p=0.012)$, and the annual mean cost/unit was $€ 2090$ vs $€ 2513$, respectively $(\mathrm{p}<0.001)$. The number of exacerbations was directly correlated with $\operatorname{FEV}_{1}(\beta=-0.157)$, age $(\beta=0.071)$, Charlson index $(\beta=0.050)$ and device type (extrafine: $\beta=0.049)(p<0.02)$.

Conclusion: In patients with COPD receiving multi-inhaler triple therapy, higher ICS doses were not associated with a further reduction in exacerbations, whereas we found an increased risk of pneumonia. The use of inhaler devices delivering extrafine ICS particle was associated with a lower rate of exacerbations, resulting in lower overall HCRU costs.

Keywords: COPD, triple-therapy, extrafine particle, exacerbations, pneumonia, health costs

\section{Introduction}

Chronic obstructive pulmonary disease (COPD) is characterized by progressive, difficult-to-reverse, chronic limitation of the airflow ${ }^{1}$ that is associated with an abnormal inflammatory lung response to harmful particles or gases, especially tobacco, with significant systemic repercussions. ${ }^{1,2}$ Around the world, COPD is an increasingly prevalent disease, ${ }^{3,4}$ with a prevalence of $6-10.4 \%$ estimated in Spain, among people aged $40-80$ years. ${ }^{4}$ COPD is a public health problem as it is the fourth leading cause of death worldwide, and one of the leading causes of morbidity, occupational disability, and worsened quality of life. ${ }^{1,2}$ COPD results in

Correspondence: Antoni Sicras-Mainar Atrys Health SA, C/Provença 392, bajos, Barcelona 08025 Spain

$\mathrm{Tel}+34934581561$

Email ansicras@atryshealth.com

International Journal of Chronic Obstructive Pulmonary Disease 2020:I5 329|-3302 
high healthcare costs, with the most severe patients and COPD exacerbations consuming the most resources. ${ }^{1-3}$ In Spain, the direct mean cost per patient with COPD is estimated at $€ 1712-3238 /$ year, $50-70 \%$ of which are in hospital costs. ${ }^{5-7}$

Maintenance pharmacotherapy for COPD is based on long-acting bronchodilators as the first line of treatment with long-acting beta adrenergic [LABA] and/or longacting anticholinergics [LAMA]. LABA in combination with inhaled corticosteroid (ICS) is reserved for patients with frequent exacerbations and/or a mixed COPD-asthma phenotype, regardless of the level of functional severity, ${ }^{1,8,9}$ and triple therapy with LAMA-LABA-ICS can be a potentially effective treatment in this group of patients as well. ${ }^{2,9}$ The TRILOGY ${ }^{10}$ and FULFIL ${ }^{11}$ clinical trials highlighted the superiority of the combination of the three drugs in patients with severe COPD over the use of IC/LABA alone. In clinical practice, the use of ICS in COPD is controversial (balance between efficacy and safety [pneumonia]), with arguments both for and against their use, and only in combination with an LABD. ${ }^{12}$ The WISDOM study ${ }^{13,14}$ found no increased risk of adverse effects in triple therapy compared with dual bronchodilation (LABA/ LAMA). However, the benefit-risk ratio of ICS-containing therapy may depend on several factors, including the ICS molecule characteristics (potency, lipophilicity), dose, and particle size. ${ }^{9,12}$ There seems to be some consensus on the use of medium-low dose ICS in patients with the COPDasthma phenotype and in frequent exacerbators. ${ }^{12}$

Particle size is the major determinant in the deposition and distribution of inhaled drug within the lungs and hence is related to local efficacy. The particle size distribution of an aerosol is usually expressed in terms of its mass median aerodynamic diameter (MMAD). To reach the lower respiratory tract past the carina, the MMAD of inhaled particles should be less than $5 \mu \mathrm{m}$ in diameter; specifically, the particle size with the most efficient deposition in the small airways, so-called extrafine particle fraction, is said to be less than $2 \mu \mathrm{m} .{ }^{15}$ The literature reviewed does not usually differentiate between the type of particles (extrafine vs non-extrafine/fine) contained in inhalers used for treating COPD. ${ }^{16}$ While most ICS are fine particles with an MMAD of 2-4 microns, some presentations use extrafine particles (approximate diameter of 1 micron). Some authors suggest there may be better effectiveness and clinical safety with the use of extrafine particle devices for the treatment of COPD, ${ }^{17,18}$ but there is very little data on their impact in normal clinical practice. In addition, evidence on the healthcare resource use and associated costs in this patient group is limited. The objective of the study was to determine the clinical (exacerbations and pneumonia) and economic consequences (healthcare resource use and costs) of the use of different doses of inhaled corticosteroids (high/medium/ low) and their particle size (extrafine vs non-extrafine) in multi-inhaler triple inhalation therapy for COPD.

\section{Patients and Methods Design and Study Population}

An observational, multicentre, longitudinal retrospective study was carried out using a before/after design with a control group, made by review of computerized medical records. The study population was obtained from the records of health providers from various centres in Spain (unified in the dissociated BIG-PAC ${ }^{\circledR}$ database; Real Life Data; http://www.encepp.eu/encepp/search.htm). The medical records come from different primary care centers and hospitals (integrated areas) from seven Autonomous Communities of the Spanish territory (computerized medical records, 1.9 million patients). Data are subject to rigorous validation and recoding (anonymized/dissociated data) and are then exported to the BIG-PAC ${ }^{\circledR}$ database. The population assigned to the participating centres is mostly urban with medium-low socioeconomic status.

\section{Inclusion and Exclusion Criteria}

Patients who sought care and initiated treatment with multi-inhaler triple therapy (LAMA-LABA-ICS) between 1 January 2015 and 31 March 2017 were included in the study. Inclusion criteria were: (a) age $\geq 40$ years, (b) date of diagnosis of $\mathrm{COPD} \geq 12$ months before the index date, (c) inclusion in the prescription programme for obtaining medical prescriptions (with a recorded daily dose, time interval and duration of each treatment administered, and two or more prescriptions), (d) guaranteed regular monitoring (two health records in the computer system) and (e) $\geq 6$ months on treatment with triple therapy. Exclusion criteria were (a) transfer out to other centres, displaced or out-of-are, (b) permanent institutionalization, and (c) a history of pulmonary emphysema, bronchiectasis, cystic fibrosis and/or bronchial neoplasm.

\section{Study Groups and Follow-Up Period}

Patients were differentiated according to: 1) ICS dose: a) low dose, b) medium dose and c) high dose; and 2) ICS 
particle size: a) extrafine particle (EFP) $\left(\right.$ Modulite $^{\circledR}$, NextHaler ${ }^{\mathbb{B}}$ ) and b) non-extrafine particle (nEFP). Patients were classified in ICS dose groups following the Bassan ${ }^{18}$ criteria according to the initial prescription regimen (Supplementary Table 2). The follow-up period was 1 year from the index date. Analysis of the ICS-containing inhalers excluded patients receiving high doses of ICS $(\mathrm{N}=724)$, as patients were not receiving high doses of ICS in extrafine particle devices for comparison. ${ }^{18}$

\section{Definition of COPD, Exacerbation, and Pneumonia}

Records of patients with COPD were obtained using the International Classification of Diseases (ninth edition) Clinical Modification (ICD-9-MC; codes 491, 492 and/or 496 for COPD and/or exacerbations) or their ICD-10 equivalents (codes: J41, J42, J44). The physician's judgement was the criteria followed. However, confirmation of the COPD diagnosis required the recording of no significant reversibility airflow obstruction following bronchodilator administration according to forced spirometry and the forced volume in the first second $\left(\mathrm{FEV}_{1} /\right.$ forced vital capacity ratio $<70 \%$ in the stable phase). The Global Initiative for Obstructive Lung Disease (GOLD) ${ }^{8}$ guidelines, which define an exacerbation as an event in the natural course of the disease characterized by a change in patient's baseline dyspnoea, cough and/or sputum that goes beyond daily variations, which is acute in its onset, and may require a change in regular medication (sustained aggravation of the baseline situation). In this respect, two types of exacerbations were considered: (a) severe: those requiring hospital admission, and (b) moderate: those requiring treatment with antibiotics and/or oral corticosteroids. For each exacerbation, blood eosinophil levels and the time from diagnosis (in years) were obtained. In addition, lung function $\left(\mathrm{FEV}_{1}\right)$ and the functional severity of COPD (classification) were also obtained according to $\mathrm{GOLD}^{8}$ recommendations. Additionally, the records of patients with pneumonia were obtained using ICD-9-MC; codes $482-486$, or their equivalents, according to ICD10: J12-J18.

\section{Sociodemographic and Comorbidity Variables}

Sociodemographic and comorbidity variables recorded were age (continuous and by intervals), sex, and the history of hypertension, diabetes, dyslipidaemia, obesity (BMI $30 \mathrm{~kg} / \mathrm{m}^{2}$ ), active smoking, ischemic heart disease, stroke, heart failure and other conditions (Table 1). As a summary variable of general comorbidity, a) the Charlson comorbidity index ${ }^{19}$ and b) the number of chronic comorbidities were collected for each patient.

\section{Description of Treatment}

The medications indicated for the treatment of COPD were obtained according to the Anatomical Therapeutic Chemical Classification System (ATC): ${ }^{20}$ oral corticosteroids (OCS, H02AB), IC/LABA (R03AK), beta-2 short-acting agonists (SABA, R03AC), short-acting muscarinic antagonist or anticholinergics (SAMA, R03BB), LAMA (R03BB), xanthines (R03DA and R03DB), leukotriene receptor antagonists (R03DC), and home oxygen. In addition, patients who received treatment with long-acting oral/systemic corticosteroids (chronic schedule) were differentiated from those who received them only for the control of an exacerbation. The duration of ICS treatment was calculated as the difference in dates between the initial prescription (index date) and the final prescription (last available prescription), in months (during the follow-up period). In addition, a treatment change was considered when there was a modification of any of the three molecules (ICS, LAMA, LABA), with respect to the baseline situation. A change in treatment was not considered when there was a variation in the dose or the type of device used.

\section{Healthcare Resource Use}

Direct health costs related to care (medical visits, days of hospitalization, emergency visits, diagnostic or therapeutic requests and medication) provided by professionals were collected. Costs were expressed at the mean cost per patient per year (mean PPY). The study concepts and their costs in $€ 2018$ are shown in Supplementary Table 1. The cost of prescriptions for each medication was quantified according to the retail price per pack at the time of prescription.

\section{Ethical and Legal Aspects}

Clinical records in the BIG-PAC database are fully anonymised, and researchers do not have access to patients' personal data. When the information used does not contain personal data, Spanish law exempts studies from evaluation by a Research Ethics Committee.

\section{Statistical Analysis}

Data were validated to ensure the quality of the records. A descriptive-univariate statistical analysis was made. 
Qualitative data were expressed as absolute and relative frequencies and quantitative data as means and standard deviation or medians and interquartile ranges. The $95 \%$ confidence intervals (CI) used to estimate parameters were based on the total number of subjects without missing values. The normality of distribution was tested using the Kolmogorov-
Smirnov test. In the bivariate analysis, ANOVA, the chisquare and Pearson's linear correlation tests were used.

The multivariate models used were (a) covariance analysis (ANCOVA; procedure: estimation of marginal means; Bonferroni's adjustment) for correction of resource use (dependent variable, according to patient classification),

Table I Baseline Characteristics of Study Subjects, COPD Classification and Medication Administered During Follow-Up by Study Groups

\begin{tabular}{|c|c|c|c|c|c|}
\hline Inhaled ICS Doses & Low & Medium & High & Total & $\mathbf{p}$ \\
\hline Number of Patients, \% & $N=849(38.9 \%)$ & $\mathrm{N}=6 \mid 2(28.0 \%)$ & $N=724(33.1 \%)$ & $N=2185(100 \%)$ & \\
\hline $\begin{array}{l}\text { Sociodemographic features } \\
\text { Mean age, years }\end{array}$ & $71.6(11.8)$ & $72.5(11.4)$ & $72.8(10.5)$ & $72.3(11.3)$ & 0.094 \\
\hline $\begin{array}{l}\text { Age Range, years } \\
40-64 \\
65-74 \\
\geq 75 \\
\text { Sex (male) }\end{array}$ & $\begin{array}{l}27.3 \% \\
29.2 \% \\
43.5 \% \\
80.7 \%\end{array}$ & $\begin{array}{l}23.5 \% \\
28.9 \% \\
47.5 \% \\
82.4 \%\end{array}$ & $\begin{array}{l}21.3 \% \\
29.0 \% \\
49.7 \% \\
80.4 \%\end{array}$ & $\begin{array}{l}24.3 \% \\
29.1 \% \\
46.7 \% \\
81.1 \%\end{array}$ & $\begin{array}{l}0.049 \\
0.619\end{array}$ \\
\hline $\begin{array}{l}\text { Overall comorbidity } \\
\text { Mean of diagnoses } \\
\text { Mean Charlson Index } \\
\text { I } \\
2 \\
3+\end{array}$ & $\begin{array}{l}3.6(1.9) \\
1.6(1.5) \\
32.0 \% \\
19.1 \% \\
24.6 \%\end{array}$ & $\begin{array}{l}3.7(1.9) \\
1.8(1.5) \\
30.4 \% \\
21.4 \% \\
26.5 \%\end{array}$ & $\begin{array}{l}3.7(1.9) \\
1.7(1.5) \\
30.7 \% \\
19.3 \% \\
27.5 \%\end{array}$ & $\begin{array}{l}3.7(1.9) \\
1.7(1.5) \\
31.1 \% \\
19.8 \% \\
26.1 \%\end{array}$ & $\begin{array}{l}0.413 \\
0.332 \\
0.334\end{array}$ \\
\hline $\begin{array}{l}\text { Associated comorbidities } \\
\text { Hypertension } \\
\text { Diabetes mellitus } \\
\text { Dyslipidaemia } \\
\text { Obesity } \\
\text { Active smoker } \\
\text { Ischemic heart disease } \\
\text { Cerebrovascular accident } \\
\text { Heart failure } \\
\text { Kidney failure } \\
\text { Depressive syndrome } \\
\text { Malign neoplasm } \\
\text { Asthma }\end{array}$ & $\begin{array}{l}60.1 \% \\
26.3 \% \\
55.0 \% \\
33.7 \% \\
23.1 \% \\
16.5 \% \\
11.2 \% \\
23.2 \% \\
10.5 \% \\
13.3 \% \\
14.1 \% \\
13.0 \%\end{array}$ & $\begin{array}{l}61.6 \% \\
28.1 \% \\
51.1 \% \\
33.5 \% \\
23.2 \% \\
19.8 \% \\
13.1 \% \\
27.1 \% \\
11.3 \% \\
15.2 \% \\
14.2 \% \\
11.4 \%\end{array}$ & $\begin{array}{l}61.9 \% \\
27.1 \% \\
55.1 \% \\
34.0 \% \\
21.0 \% \\
17.8 \% \\
11.0 \% \\
24.0 \% \\
11.6 \% \\
13.3 \% \\
15.3 \% \\
12.7 \%\end{array}$ & $\begin{array}{l}61.1 \% \\
27.0 \% \\
54.0 \% \\
33.7 \% \\
22.4 \% \\
17.8 \% \\
11.7 \% \\
24.6 \% \\
11.1 \% \\
13.8 \% \\
14.6 \% \\
12.4 \%\end{array}$ & $\begin{array}{l}0.731 \\
0.737 \\
0.258 \\
0.982 \\
0.528 \\
0.271 \\
0.443 \\
0.210 \\
0.767 \\
0.509 \\
0.768 \\
0.664\end{array}$ \\
\hline $\begin{array}{l}\text { COPD Severity } \\
\text { GOLD II (moderate) } \\
\text { GOLD III (severe) } \\
\text { GOLD IV (very severe) }\end{array}$ & $\begin{array}{l}64.8 \% \\
34.0 \% \\
1.2 \%\end{array}$ & $\begin{array}{l}57.8 \% \\
37.2 \% \\
2.0 \%\end{array}$ & $\begin{array}{l}59.7 \% \\
42.8 \% \\
2.5 \%\end{array}$ & $\begin{array}{l}61.1 \% \\
37.0 \% \\
1.8 \%\end{array}$ & 0.029 \\
\hline $\begin{array}{l}\text { Other parameters/variables } \\
\text { Time from diagnosis, years } \\
\mathrm{BMI}, \mathrm{kg} / \mathrm{m}^{2} \\
\mathrm{FEV}, \mathrm{l} \\
\mathrm{Blood} \text { eosinophils, cells } / \mu \mathrm{L} \\
\text { Mean ICS dose }\end{array}$ & $\begin{array}{l}15.3(6.4) \\
29.1(5.2) \\
60.7(13.2) \\
185(I 12) \\
284.5(83.1)\end{array}$ & $\begin{array}{l}15.9(6.0) \\
29.1(5.0) \\
58.6(13.5) \\
186(125) \\
475.5(67.1)\end{array}$ & $\begin{array}{l}16.3(5.8) \\
28.7(4.8) \\
54.9(14.3) \\
182(103) \\
986.7(121.5)\end{array}$ & $\begin{array}{l}15.8(6.1) \\
29.0(5.0) \\
58.2(13.9) \\
184(119) \\
570.7(317.1)\end{array}$ & $\begin{array}{l}0.011 \\
0.229 \\
<0.001 \\
0.527 \\
0.001\end{array}$ \\
\hline
\end{tabular}

Notes: Values expressed as percentage or mean (SD: standard deviation), p: statistical significance.

Abbreviations: ICS, inhaled corticosteroids; GOLD, Global Initiative for Chronic Obstructive Lung Disease; BMI, body mass index, kg/m²; FEV in the first second. 
and (b) multiple linear regression to determine variables associated with health costs and exacerbations (procedure: consecutive steps). The covariates included in the models were age, sex, $\mathrm{FEV}_{1}$, the Charlson index and time from diagnosis. Statistical significance was established as $\mathrm{p}<0.05$. The analysis was made using SPSSWIN version 23 .

\section{Results}

Of an initial population of 1.9 million, 802,237 patients aged $\geq 40$ years received care between 1 January 2015 and 31 March 2017. Of these, 13,690 patients were diagnosed with COPD. Finally, 2185 patients who met the inclusion/ exclusion criteria were analysed and followed during the study period (Figure 1).

\section{Results According to ICS Dose}

Table 1 shows the general characteristics of patients according to study groups: 849 patients received low-dose ICS (38.9\%), 612 received medium-dose ICS (28.0\%) and 724 received high-dose ICS (33.1\%). The distribution of ICS dose according to the different drug combinations (number of patients) is detailed in Supplementary Table S3. The mean age was 72.3 years and $81.1 \%$ were male. Hypertension $(61.1 \%)$, dyslipidaemia (54\%), obesity (33.7\%) and diabetes $(27 \%)$ were the most frequent comorbidities. There was acceptable comparability between the ICS dose groups with respect to sociodemographic characteristics and associated comorbidities.
Table 2 shows the main complications (exacerbations and pneumonia) according to the ICS dose. Total exacerbations increased from baseline with increasing dose of ICS (low: 26.4\%, medium: 28.7\% and high: $30.4 \%(p=0.047))$. Both moderate $(26.2 \%, 28.9 \%$ and $29.1 \% \quad(\mathrm{p}=0.044))$ and severe exacerbations $(6.7 \%$, $9.2 \%$ and $11.2 \%(\mathrm{p}=0.014))$ were higher in patients on high-dose IC. The mean time to first exacerbation was significantly shorter in patients taking high doses of ICS (132, 130 and 116 days, respectively $(\mathrm{p}<0.05)$ ) (Table 2). Significant associations were also observed between high doses and the functional severity of COPD (in severe COPD; low: 34\%, medium: 37.2\%, and high: $42.8 \%(\mathrm{p}=0.029))$, as well as between ICS dose and use of oral corticosteroids (low: 21.8\%, medium: 23.5\% and high: $26.4 \%(\mathrm{p}<0.001)$ ) and \% $\mathrm{FEV}_{1}$ (low: 60.7\%; medium: 58.6\% and high: 54.9\% $(\mathrm{p}<0.001))$ (Table 2).

The proportion of pneumonia (patients) increased with the ICS dose (low: 3.4\%, medium: $4.2 \%$ and high: $6.9 \%$ $(p=0.041))$. Total healthcare costs were $€ 5.3$ million, of which $66.1 \%$ were in primary healthcare and $33.9 \%$ in specialized care. The biggest cost components were hospital admissions (9.8\%), primary healthcare visits (14\%) and medication (48.7\%). The annual mean PPPY cost (corrected for covariates, ANCOVA) was higher with increasing dose of ICS (low: $€ 2383$, medium: $€ 2401$ and high: $€ 2625$ ( $\mathrm{p}=0.024)$ ) (difference between high-/low-dose ICS: $€$ 242/patient/year) (Table 3).

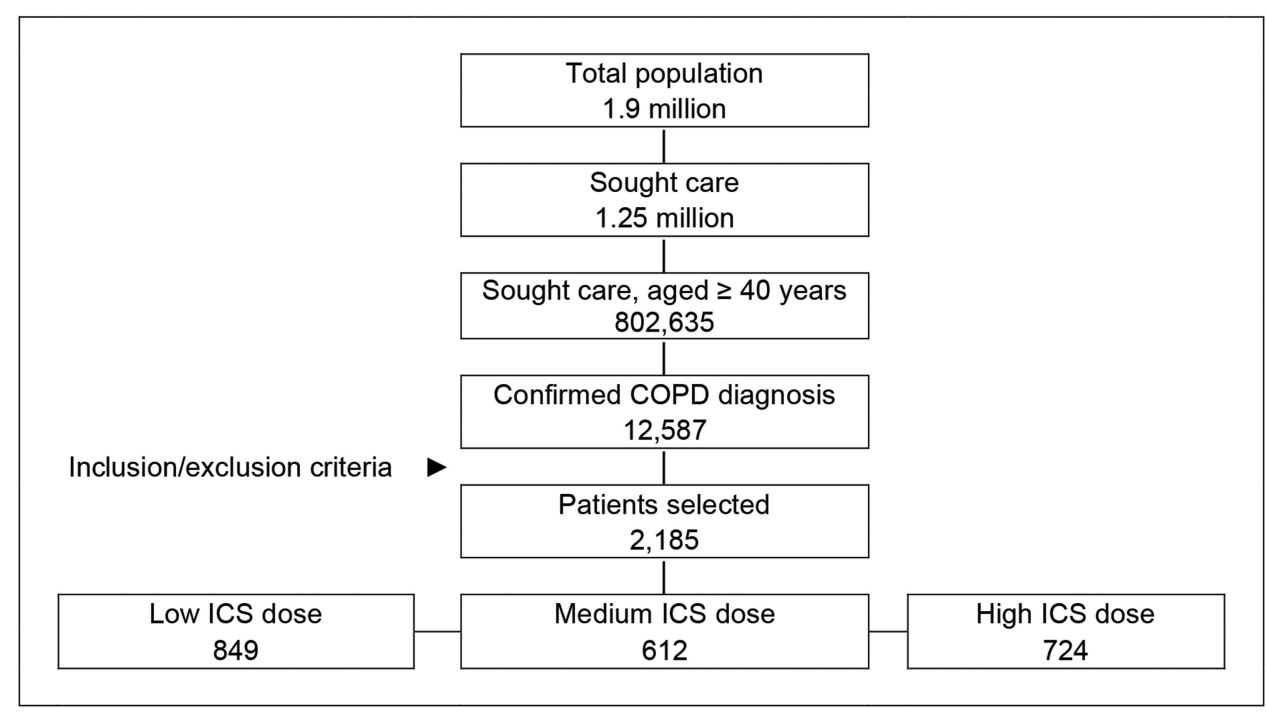

Figure I Study flow diagram. A retrospective observational study was carried out, made from the review of medical records (computerized databases, with anonymised and dissociated data) of patients diagnosed with COPD. Abbreviation: ICS, inhaled corticosteroid. 


\section{Results According to ICS Particle Size}

Table 4 shows general patient characteristics according to the particle size (subjects with high doses of ICS were excluded): 462 patients $(31.6 \%)$ received treatment with an EFP ICS/LABA, and 999 with an nEFP ICS/LABA (68.4\%). In general, the baseline characteristics were comparable between the groups analysed. Patients using EFP, compared with nEFP, had similar functional COPD severity (GOLD III: $37 \% \quad$ vs $36.4 \% ; \quad \mathrm{p}=0.861$ ), oral corticosteroid use $(26.4 \%$ vs $25.3 \%, \mathrm{p}=0.413)$ and $\mathrm{FEV}_{1}$ (59.3\% vs.60.1\%; $\mathrm{p}=0.269)$.

Table 5 shows the proportion of exacerbations and pneumonia according to the ICS particle size. Total exacerbations were lower for EFP vs $\operatorname{nEFP}(24 \%$ vs $30.4 \% ; \mathrm{p}=0.012$ ). Moderate exacerbations were also lower for EFP vs nEFP (23.6\% vs $29.9 \%$; p 0.048), while serious exacerbations (hospital admissions: 6.5\% vs $8.3 \%$; $\mathrm{p}=0.078$ ) did not differ significantly. No relevant

Table 2 Incidence of Exacerbations and Pneumonia According to Inhaled Corticosteroid (ICS) Dose

\begin{tabular}{|c|c|c|c|c|c|}
\hline Inhaled ICS Doses & Low & Medium & High & Total & \multirow{2}{*}{$\mathbf{p}$} \\
\hline Number of Patients & $N=849$ & $N=6 / 2$ & $N=724$ & $N=2185$ & \\
\hline \multicolumn{6}{|l|}{ Follow-up period (one year) } \\
\hline Mean (SD) number of exacerbations & $0.5(1.0)$ & $0.6(1.2)$ & $0.6(1.2)$ & $0.6(I . I)$ & 0.062 \\
\hline \% Patients with 0 exacerbation & $72.6 \%$ & $70.3 \%$ & $69.6 \%$ & $70.9 \%$ & \\
\hline$\%$ Patients with $\geq 1$ exacerbation & $26.4 \%$ & $28.7 \%$ & $30.4 \%$ & $29.0 \%$ & 0.047 \\
\hline 1 & $16.5 \%$ & $16.7 \%$ & $18.4 \%$ & $17.2 \%$ & \\
\hline 2 & $6.1 \%$ & $4.4 \%$ & $4.6 \%$ & $5.1 \%$ & \\
\hline $3+$ & $4.8 \%$ & $8.7 \%$ & $7.5 \%$ & $6.8 \%$ & 0.024 \\
\hline Mean nb (SD) number of mod exacerbation & $0.4(0.8)$ & $0.5(0.9)$ & $0.5(1.0)$ & $0.4(0.9)$ & 0.257 \\
\hline \% Patients with 0 mod exacerbation & $72.8 \%$ & $71.1 \%$ & $70.9 \%$ & $71.7 \%$ & \\
\hline$\%$ Patients with $\geq 1$ mod exacerbation & $26.2 \%$ & $28.9 \%$ & $29.1 \%$ & $28.3 \%$ & 0.044 \\
\hline 1 & $19.4 \%$ & $19.0 \%$ & $21.3 \%$ & $19.9 \%$ & \\
\hline 2 & $3.8 \%$ & $4.1 \%$ & $1.7 \%$ & $3.2 \%$ & \\
\hline $3+$ & $4.0 \%$ & $5.9 \%$ & $6.2 \%$ & $5.3 \%$ & 0.149 \\
\hline Mean nb (SD) of severe exacerbation & $0.1(0.3)$ & $0.1(0.4)$ & $0.1(0.4)$ & $0.1(0.4)$ & 0.003 \\
\hline$\%$ Patients with 0 severe exacerbation & $93.3 \%$ & $90.8 \%$ & $88.8 \%$ & $91.1 \%$ & \\
\hline$\%$ Patients with $\geq 1$ severe exacerbation & $6.7 \%$ & $9.2 \%$ & $11.2 \%$ & $8.9 \%$ & 0.014 \\
\hline I & $6.0 \%$ & $7.2 \%$ & $8.8 \%$ & $7.3 \%$ & \\
\hline 2 & $0.7 \%$ & $2.0 \%$ & $2.3 \%$ & $1.6 \%$ & 0.014 \\
\hline \multicolumn{6}{|l|}{ Time to first exacerbation, days } \\
\hline Mean (SD) & $142.6(98.9)$ & $140.3(95.1)$ & $123.8(82)$ & $135.5(92.5)$ & 0.047 \\
\hline Median (P25 - P75) & $132(\mid 12-152)$ & $130(|1|-149)$ & $116(99-133)$ & $130(|19-14|)$ & \\
\hline Mean (SD) nb of pneumonias & $0.0(0.2)$ & $0.0(0.2)$ & $0.1(0.3)$ & $0.1(0.2)$ & 0.002 \\
\hline \% Patients with pneumonia & $3.4 \%$ & $4.2 \%$ & $6.9 \%$ & $4.7 \%$ & 0.041 \\
\hline Death (\% patients) & $9.8 \%$ & $11.4 \%$ & $12.4 \%$ & $11.1 \%$ & 0.238 \\
\hline \multicolumn{6}{|l|}{ Medication use (\% patients) } \\
\hline Oral corticosteroids & $21.8 \%$ & $23.5 \%$ & $26.4 \%$ & $25.3 \%$ & $<0.001$ \\
\hline Chronic use of oral corticosteroids & $5.2 \%$ & $7.5 \%$ & $10.9 \%$ & $8.1 \%$ & 0.044 \\
\hline Systemic antibiotics & $22.5 \%$ & $25.7 \%$ & $27.9 \%$ & $23.8 \%$ & 0.020 \\
\hline Beta-2 short-acting agonists & $94.0 \%$ & $92.0 \%$ & $92.3 \%$ & $92.9 \%$ & 0.256 \\
\hline Xanthines & $9.9 \%$ & $8.7 \%$ & $9.5 \%$ & $9.4 \%$ & 0.723 \\
\hline Leukotriene receptor antagonists & $2.0 \%$ & $2.1 \%$ & $1.7 \%$ & $1.9 \%$ & 0.806 \\
\hline Home oxygen therapy & $11.5 \%$ & $11.4 \%$ & $14.0 \%$ & $12.3 \%$ & 0.259 \\
\hline Duration of treatment (ICS), in months & $10.3(2.3)$ & $10.5(2.4)$ & $10.1(2.2)$ & $10.4(2.3)$ & 0.748 \\
\hline Treatment changes & $1.9(0.2)$ & $1.8(0.2)$ & $1.9(0.2)$ & $1.9(0.2)$ & 0.681 \\
\hline
\end{tabular}

Note: Values expressed as percentage or mean.

Abbreviations: SD, standard deviation; p, statistical significance; P, percentile; IC, inhaled corticosteroids. 
Table 3 Resource Use and Costs by Study Groups

\begin{tabular}{|c|c|c|c|c|c|}
\hline Inhaled ICS Doses & Low & Medium & High & Total & \multirow[t]{2}{*}{$\mathbf{p}$} \\
\hline Number of Patients, \% & $N=849$ & $N=612$ & $N=724$ & $N=2185$ & \\
\hline \multicolumn{6}{|l|}{ Resource use } \\
\hline Medical visits, primary care & I4.5 (9.I) & $15.3(10.1)$ & $15.5(10.9)$ & $15.0(10.0)$ & 0.133 \\
\hline Laboratory tests & $0.5(0.9)$ & $0.6(1.0)$ & $0.7(\mathrm{I} . \mathrm{I})$ & $0.6(1.0)$ & 0.007 \\
\hline Conventional radiology & $0.4(1.2)$ & $0.6(1.7)$ & $0.6(1.6)$ & $0.5(1.5)$ & 0.015 \\
\hline Complementary tests & $0.4(1.3)$ & $0.4(1.0)$ & $0.5(\mathrm{I} .3)$ & $0.4(\mathrm{I} .2)$ & 0.466 \\
\hline Days of hospitalization & $0.1(0.3)$ & $0.1(0.4)$ & $0.1(0.4)$ & $0.1(0.3)$ & 0.013 \\
\hline Medical visits, hospitals & $3.5(3.1)$ & $3.8(3.5)$ & $3.9(3.3)$ & $3.7(3.3)$ & 0.030 \\
\hline Emergency visits, hospitals & $2.7(2.0)$ & $3(2.2)$ & $3.0(2.2)$ & $2.9(2.1)$ & 0.001 \\
\hline \multicolumn{6}{|l|}{ Gross costs $(€)$} \\
\hline Costs in primary care & $156 \mid(536)$ & I569 (59I) & $\mid 757(63 \mid)$ & $1628(59 \mid)$ & $<0.001$ \\
\hline Medical visits & $334(210)$ & $35 I(232)$ & $356(250)$ & $346(230)$ & 0.133 \\
\hline Laboratory tests & $19(32)$ & $21(34)$ & $24(37)$ & $21(34)$ & 0.007 \\
\hline Conventional radiology & $19(58)$ & $30(78)$ & $26(76)$ & $24(70)$ & 0.015 \\
\hline Complementary tests & $36(108)$ & $34(86)$ & $4 I(I I 3)$ & $37(104)$ & 0.466 \\
\hline Medications & II54 (397) & $1133(391)$ & $1309(435)$ & $1199(416)$ & 0.001 \\
\hline Costs, specialized care & $730(985)$ & $887(1249)$ & 914 (1229) & $835(1149)$ & 0.003 \\
\hline Days of hospitalization & $179(701)$ & $268(907)$ & $298(908)$ & $243(835)$ & 0.013 \\
\hline Medical visits & $236(2 \mid 2)$ & $262(235)$ & $263(222)$ & $252(222)$ & 0.030 \\
\hline Emergency visits & $315(237)$ & $357(261)$ & $354(256)$ & $339(25 \mathrm{I})$ & 0.001 \\
\hline Health costs & $2291(1313)$ & $2456(1674)$ & $2670(1670)$ & $2.463(1550)$ & $<0.001$ \\
\hline Corrected cost model $(€)^{*}$ & & & & Difference & \\
\hline Costs in primary care & 1573 & 1558 & 1749 & 177 & $<0.001$ \\
\hline $95 \% \mathrm{Cl}$ & $1522-1622$ & $1497-16 \mid 8$ & $1695-1803$ & & \\
\hline Costs in specialized care & 811 & 843 & 876 & 65 & 0.654 \\
\hline $95 \% \mathrm{Cl}$ & $717-904$ & $729-956$ & 774-977 & & \\
\hline Total health costs & 2383 & 2401 & 2625 & 242 & 0.024 \\
\hline $95 \% \mathrm{Ci}$ & $2256-2510$ & $2247-2554$ & $2488-2761$ & & \\
\hline
\end{tabular}

Notes: Values expressed as mean. *ANCOVA model: Contrasts are based on pairwise comparisons between estimated marginal means.

Abbreviations: SD, standard deviation; p, statistical significance; $\mathrm{Cl}$, confidence interval; IC, inhaled corticosteroids.

differences were found according to ICS duration or treatment changes (ICS, LABA, LAMA) across the different cohorts analyzed, during the follow-up period (Tables 1 and 5). The incidence of pneumonia was similar between the two groups ( $3 \%$ vs. $4.3 \% ; \mathrm{p}=0.378)$. For $n E F P$, the median time to first exacerbation was faster than for EFP (126 vs.160 days $(p=0.004)$ ). The annual mean PPPY healthcare cost with an EFP ICS was lower than that on nEFP ICS $(€ 2090$ vs $€ 2,513(\mathrm{p}<0.00))$ (difference: $€$ $-423 /$ patient/year) (Table 6).

\section{Joint Analysis}

In the binary correlation model, age was principally associated with the time from diagnosis $(\mathrm{r}=0.332)$, comorbidity $(\mathrm{r}=0.338)$, mortality $(\mathrm{r}=0.258)$ and exacerbations $(\mathrm{r}=0.177)$. Comorbidity was associated with healthcare costs $(r=0.162)$, exacerbations $(r=0.138)$ and pneumonia $(r=0.075)$. High doses of ICS were associated with $\mathrm{FEV}_{1}$ $(\mathrm{r}=0.178)$, and healthcare costs with exacerbations $(\mathrm{r}=0.792)$ and pneumonia $(\mathrm{r}=0.145)$. nEFP ICS were associated with the number of exacerbations $(r=0.077)$ and healthcare costs $(r=0.154)(\mathrm{p}<0.03)$. In the multiple linear regression analysis, healthcare costs were associated with the number of exacerbations $(\beta=0.807)$, age $(\beta=0.178)$, ICS particle size (nEFP: $\beta=0.121$ ), high doses of ICS $(\beta=0.042)$, comorbidity $(\beta=0.041)$ and $\mathrm{FEV}_{1}(\beta=-0.024)$ $(p<0.02$ in all cases). The model determination coefficient was $65.2 \%$. The number of exacerbations (during followup) was associated with $\mathrm{FEV}_{1}(\beta=-0.157)$, age $(\beta=0.071)$, comorbidity (Charlson: $\beta=0.050$ ) and ICS particle size 
Table 4 Baseline Characteristics of Patients by Study Groups

\begin{tabular}{|c|c|c|c|}
\hline Type of Particles & Extrafine & Non-Extrafine & \multirow[t]{2}{*}{$\mathbf{p}$} \\
\hline Number of Patients, \% & $\mathrm{N}=462(31.6 \%)$ & $\mathrm{N}=999(68.4 \%)$ & \\
\hline \multicolumn{4}{|l|}{ Sociodemographic features } \\
\hline Mean age, years & $72.5(11.5)$ & $71.8(11.7)$ & 0.301 \\
\hline Range: $40-64$ years & $25.3 \%$ & $25.9 \%$ & \\
\hline $65-74$ years & $26.8 \%$ & $30.1 \%$ & \\
\hline$\geq 75$ years & $47.8 \%$ & $43.9 \%$ & 0.320 \\
\hline Sex (male) & $81.4 \%$ & $81.4 \%$ & 0.999 \\
\hline \multicolumn{4}{|l|}{ Overall comorbidity } \\
\hline Mean diagnoses & $3.6(1.8)$ & $3.7(1.9)$ & 0.521 \\
\hline Mean Charlson Index & $1.7(1.5)$ & $1.7(1.5)$ & 0.955 \\
\hline 1 & $33.8 \%$ & $30.2 \%$ & \\
\hline 2 & $19.7 \%$ & $20.2 \%$ & \\
\hline $3+$ & $24.9 \%$ & $25.6 \%$ & 0.367 \\
\hline \multicolumn{4}{|l|}{ Associated comorbidities } \\
\hline Hypertension & $62.3 \%$ & $60.0 \%$ & 0.387 \\
\hline Diabetes mellitus & $27.1 \%$ & $27.0 \%$ & 0.991 \\
\hline Dyslipidaemia & $55.4 \%$ & $52.5 \%$ & 0.292 \\
\hline Obesity & $34.2 \%$ & $33.3 \%$ & 0.745 \\
\hline Active smoker & $22.9 \%$ & $23.2 \%$ & 0.906 \\
\hline Ischemic heart disease & $17.1 \%$ & $18.2 \%$ & 0.604 \\
\hline Cerebrovascular accident & $11.7 \%$ & $12.1 \%$ & 0.817 \\
\hline Heart failure & $24.9 \%$ & $24.8 \%$ & 0.978 \\
\hline Kidney failure & $10.2 \%$ & $11.1 \%$ & 0.591 \\
\hline Depressive syndrome & $13.9 \%$ & $14.2 \%$ & 0.854 \\
\hline Malign neoplasm & $14.5 \%$ & $14.0 \%$ & 0.804 \\
\hline Asthma & $14.7 \%$ & $11.2 \%$ & 0.058 \\
\hline \multicolumn{4}{|l|}{ COPD Severity } \\
\hline GOLD II (moderate) & $61.3 \%$ & $62.2 \%$ & \\
\hline GOLD III (severe) & $37.0 \%$ & $36.4 \%$ & \\
\hline GOLD IV (very severe) & $1.7 \%$ & $1.4 \%$ & 0.861 \\
\hline \multicolumn{4}{|l|}{ Other parameters/variables } \\
\hline Time from diagnosis, years & $16(6.3)$ & $15.4(6.0)$ & 0.083 \\
\hline BMI, $\mathrm{kg} / \mathrm{m}^{2}$ & $29.3(5.2)$ & $29(5.1)$ & 0.385 \\
\hline $\mathrm{FEV}_{1}, \%$ & $59.3(13.3)$ & $60.1(13.4)$ & 0.269 \\
\hline Blood eosinophils, cells/ $\mu \mathrm{L}$ & 181 (102.3) & $185(112.5)$ & 0.678 \\
\hline Mean ICS dose & $375.5(121.5)$ & $369.4(121.4)$ & 0.419 \\
\hline
\end{tabular}

Note: Values expressed as percentage or mean.

Abbreviations: SD, standard deviation; $p$, statistical significance; GOLD, Global Initiative for Chronic Obstructive Lung Disease; BMI, body mass index, $\mathrm{kg} / \mathrm{m}^{2} ; \mathrm{FEV}_{1}$, forced expiratory volume in the first second.

(nEFP: $\beta=0.049)(\mathrm{p}<0.02)$. The model determination coefficient was $40.1 \%$.

\section{Discussion}

The results of this study show that, in patients beginning triple therapy, an increase in the IC dose was not associated with a reduction in the number of exacerbations, while an increased risk of pneumonia was observed. Likewise, with equal severity of COPD, patients treated with EFP had lower rates of severe and moderate
Table 5 Description of Exacerbations and Pneumonia by ICS Particle Size Grouping

\begin{tabular}{|c|c|c|c|}
\hline ICS Particle Size & Extrafine & $\begin{array}{l}\text { Non- } \\
\text { Extrafine }\end{array}$ & $\mathbf{p}$ \\
\hline Number of Patients, \% & $\begin{array}{l}N=462 \\
(31.6 \%)\end{array}$ & $\begin{array}{l}N=999 \\
(68.4 \%)\end{array}$ & \\
\hline Follow-up period (one year) & & & \\
\hline Total exacerbations, \% & $24.0 \%$ & $30.4 \%$ & 0.012 \\
\hline $\begin{array}{l}\text { Mean }(S D) \text { number of } \\
\text { exacerbations }\end{array}$ & $0.5(1.1)$ & $0.5(1.1)$ & 0.172 \\
\hline$\%$ Patients with 0 exacerbation & $76.0 \%$ & $69.6 \%$ & \\
\hline I & $12.8 \%$ & $18.3 \%$ & \\
\hline 2 & $5.4 \%$ & $5.4 \%$ & \\
\hline $3+$ & $5.8 \%$ & $6.7 \%$ & 0.048 \\
\hline Moderate exacerbations, \% & $23.6 \%$ & $29.9 \%$ & 0.048 \\
\hline $\begin{array}{l}\text { Mean }(\mathrm{SD}) \text { number of } \\
\text { exacerbations }\end{array}$ & $0.4(0.9)$ & $0.5(0.9)$ & 0.165 \\
\hline$\%$ Patients with 0 exacerbation & $76.4 \%$ & $70.1 \%$ & \\
\hline 1 & $15.4 \%$ & $21.0 \%$ & \\
\hline 2 & $3.9 \%$ & $3.9 \%$ & \\
\hline $3+$ & $4.3 \%$ & $5.0 \%$ & 0.079 \\
\hline $\begin{array}{l}\text { Severe exacerbations } \\
\text { (hospitalization), \% }\end{array}$ & $6.5 \%$ & $8.3 \%$ & 0.078 \\
\hline $\begin{array}{l}\text { Mean }(\mathrm{SD}) \text { number of } \\
\text { exacerbations }\end{array}$ & $0.1(0.3)$ & $0.1(0.3)$ & 0.268 \\
\hline$\%$ Patients with 0 exacerbation & $93.5 \%$ & $91.7 \%$ & \\
\hline I & $5.4 \%$ & $7.0 \%$ & \\
\hline 2 & $1.1 \%$ & $1.3 \%$ & 0.479 \\
\hline \multicolumn{4}{|l|}{ Time to first exacerbation } \\
\hline Mean (SD) & $164.6(99)$ & I33.4 (95.2) & 0.004 \\
\hline Median (P25 - P75) & $160(138-182)$ & $126(114-138)$ & \\
\hline$\%$ Patients with pneumonia & $3.0 \%$ & $4.3 \%$ & 0.378 \\
\hline Mean (SD) nb of pneumonias & $0.0(0.2)$ & $0.1(0.2)$ & 0.102 \\
\hline Deaths & $9.7 \%$ & $10.8 \%$ & 0.534 \\
\hline \multicolumn{4}{|l|}{ Medication use } \\
\hline Oral corticosteroids & $26.4 \%$ & $25.3 \%$ & 0.413 \\
\hline Chronic oral corticosteroid use & $7.9 \%$ & $8.3 \%$ & 0.685 \\
\hline Systemic antibiotics & $21.2 \%$ & $23.2 \%$ & 0.647 \\
\hline Beta-2 short-acting agonists & $95.2 \%$ & $92.2 \%$ & 0.032 \\
\hline Xanthines & $9.7 \%$ & $9.2 \%$ & 0.746 \\
\hline Leukotriene receptor antagonists & $2.2 \%$ & $2.0 \%$ & 0.839 \\
\hline Home oxygen therapy & $11.3 \%$ & $11.6 \%$ & 0.843 \\
\hline $\begin{array}{l}\text { Duration of treatment (ICS), in } \\
\text { months }\end{array}$ & $10.5(2.2)$ & $10.1(2.3)$ & 0.799 \\
\hline Treatment changes & $1.8(0.1)$ & $1.9(0.2)$ & 0.691 \\
\hline
\end{tabular}

Note: Values expressed as percentage or mean.

Abbreviations: SD, standard deviation; $p$, statistical significance; $P$, percentile.

exacerbations, resulting in lower health costs for the Spanish National Health System.

The total percentage of exacerbations increased according to the IC dose (low: $26.4 \%$, medium: $28.7 \%$ and high: $30.4 \%)$, in line with the proportion of pneumonia $(3.4 \%$, 
Table 6 Resource Use and Costs by ICS Particle Size Grouping

\begin{tabular}{|c|c|c|c|}
\hline ICS Particle Size & Extrafine & Non-Extrafine & $\mathbf{p}$ \\
\hline Number of Patients, \% & $\begin{array}{l}N=462 \\
(31.6 \%)\end{array}$ & $\begin{array}{l}N=999 \\
(68.4 \%)\end{array}$ & \\
\hline $\begin{array}{l}\text { Resource use } \\
\text { Medical visits, primary care } \\
\text { Laboratory tests } \\
\text { Conventional radiology } \\
\text { Complementary tests } \\
\text { Days of hospitalization } \\
\text { Medical visits, hospitals } \\
\text { Emergency visits, hospitals }\end{array}$ & $\begin{array}{l}\text { I2.4(8.5) } \\
0.5(0.8) \\
0.2(0.6) \\
0.4(1.2) \\
0.1(0.3) \\
2.8(2.0) \\
2.3(1.2)\end{array}$ & $\begin{array}{l}15.9(9.8) \\
0.6(1.0) \\
0.6(1.7) \\
0.4(1.2) \\
0.1(0.3) \\
4(3.6) \\
3.1(2.4)\end{array}$ & $\begin{array}{l}<0.001 \\
0.075 \\
<0.001 \\
0.396 \\
0.315 \\
<0.001 \\
<0.001\end{array}$ \\
\hline $\begin{array}{l}\text { Gross costs }(€) \\
\text { Costs in primary care } \\
\text { Medical visits } \\
\text { Laboratory tests } \\
\text { Conventional radiology } \\
\text { Complementary tests } \\
\text { Medications } \\
\text { Costs, specialized care } \\
\text { Days of hospitalization } \\
\text { Medical visits } \\
\text { Emergency visits } \\
\text { Health costs }\end{array}$ & $\begin{array}{l}1368(500) \\
285(196) \\
18(28) \\
9(28) \\
32(101) \\
1025(382) \\
643(905) \\
186(743) \\
190(136) \\
267(137) \\
2011(1185)\end{array}$ & $\begin{array}{l}1655(562) \\
366(224) \\
21(34) \\
30(78) \\
36(98) \\
1200(388) \\
867(1180) \\
230(817) \\
273(247) \\
364(280) \\
2522(1568)\end{array}$ & $\begin{array}{l}<0.001 \\
<0.001 \\
0.075 \\
<0.001 \\
0.396 \\
<0.001 \\
<0.001 \\
0.315 \\
<0.001 \\
<0.001 \\
<0.001\end{array}$ \\
\hline $\begin{array}{l}\text { Corrected cost model }(€)^{*} \\
\text { Costs in primary care } \\
95 \% \mathrm{Cl} \\
\text { Costs in specialized care } \\
95 \% \mathrm{Cl} \\
\mathrm{Health} \text { costs } \\
95 \% \mathrm{Cl}\end{array}$ & $\begin{array}{l}1376 \\
1313-1438 \\
714 \\
591-837 \\
2090 \\
1926-2252\end{array}$ & $\begin{array}{l}1657 \\
1614-1700 \\
856 \\
771-939 \\
2513 \\
2401-2624\end{array}$ & $\begin{array}{l}<0.001 \\
0.061 \\
<0.001\end{array}$ \\
\hline
\end{tabular}

Notes: Values expressed in mean. *ANCOVA model: Contrasts are based on pairwise comparisons between estimated marginal means.

Abbreviations: SD, standard deviation; $\mathrm{p}$, statistical significance; $\mathrm{Cl}$, confidence interval.

$4.2 \%$ and $6.9 \%$, respectively). The combination of IC + LAMA/LABA is controversial in the treatment of COPD. ${ }^{1,2,9,12}$ Some reviews show that triple therapy provides modest clinical benefits in the general COPD population, while achieving greater efficacy in patients with frequent exacerbations and eosinophilia (asthma-COPD phenotype). ${ }^{12,21,22}$ Conversely, adding a LAMA to an IC/ LABA combination produces significant clinical benefits in COPD patients (dual bronchodilation). ${ }^{21,22}$ Most studies highlight the discrepancies between the guideline recommendations and the therapy used in clinical practice. $9,12,21,22$

There is debate on the side effects, such as pneumonia and systemic adverse effects, of long-term IC use. Some reports suggest the safety profile of IC depends on the dose administered. Thus, there appear to be differences between the pathophysiological mechanisms related to the action of
IC, which are predominantly immunosuppressant at high doses and anti-inflammatory at lower doses. This may have practical effects, highlighting the value of low-dose IC in the treatment of COPD. ${ }^{23}$ We found that patients who received high doses of IC vs low/moderate doses showed no reduction in exacerbations, although previous exacerbations were not considered, and these patients had more severe COPD. These results show the complexity of COPD therapy, especially in severe patients. As for the risk of pneumonia, our results seem to be in line with most studies, although this topic is also controversial. Cheng ${ }^{24}$ concluded that high doses of IC had greater clinical effectiveness in COPD patients, with no higher incidence of pneumonia, and suggest that high-dose IC therapy may be appropriate in COPD patients. The Spanish PNEUMOCORT ${ }^{25}$ study found that severe pneumonia and exacerbations were more common in patients with severe COPD and in patients receiving high doses of IC, results similar to ours.

The proportion of exacerbations was $24 \%$ in patients using EFP devices and $30.4 \%$ in those using nEFP devices. In the joint analysis (IC dose vs device type), exacerbations were indirectly related to the use of EFP devices (corrected by covariates). There is little reported evidence, but the TRILOGY ${ }^{10}$ and TRINITY ${ }^{26}$ studies permit assessment of the effectiveness and safety of devices with EFP compared with other types of devices. Both studies show a slight reduction in exacerbations and the risk of pneumonia. The FORWARD ${ }^{27}$ and TRIBUTE ${ }^{28}$ studies also found a reduction in exacerbations, but no increase in the risk of pneumonia. A review by Scichilone $^{29}$ found that an inability to reach and treat peripheral airways may contribute to a lack of efficacy of inhaled treatments. The development of extrafine inhaled formulations allows a more uniform distribution of inhaled treatment throughout the respiratory tree. Better knowledge of the pathophysiology of the peripheral airways may help identify specific COPD phenotypes that would benefit from these treatments. Despite methodological discrepancies, our results may be in line with these contributions.

The mean cost per patient/year was higher in patients receiving high doses of IC compared with medium and low doses ( $€ 2625, € 2383$ and $€ 2401$, respectively). Likewise, costs were lower in patients with EFP devices ( $€$ 2090) than in those using nEFP devices ( $€$ 2513). These results are directly related to the proportion of exacerbations, especially severe exacerbations requiring hospitalization. Reviews by Press $^{30}$ and Ehteshami-Afshar ${ }^{31}$ highlighted 
the wide methodological differences in the studies analysed and concluded that reports consistently show that better disease control reduces COPD costs and that there is a growing need for economic studies based on the latest guideline recommendations. Our results are difficult to compare since we have found no similar studies in the literature search. Most studies conclude that: a) the cost is related to COPD severity, b) rehospitalizations, emergency visits and medication were the most prominent cost components, and c) increasing health education can reduce COPD costs. $^{32,33}$ In addition, COPD patients with eosinophil counts of $\geq 220$ cells $/ \mu \mathrm{L}$ are more likely to have had moderate or severe exacerbations, implying higher costs. ${ }^{34}$ Allowing for methodological limitations, our results seem to be in line with the available literature.

Our study has some limitations, principally those typical of retrospective studies, which are related to the categorization of COPD severity, the possible classification bias of patients and the limitations in the measurement of variables, attributable to the information system. In this respect, the possible inaccuracy of diagnostic coding in the diagnosis of COPD and other comorbidities, the definition of exacerbation, or the absence of variables that could influence the final results (socioeconomic level, environmental/occupational exposure, evolution of the pharmacological dose prescribed, verification of the inhalation technique, therapeutic adherence and/or the differentiation of phenotypes), should be considered as limitations. Other unmeasured factors that might have influenced the results may include: a) the length of use of IC in triple therapy, b) the pharmacological characteristics of the drug combinations, and/or c) the combination of these in each device (IC/ LAMA/LABA, IC/LABA + LAMA, LABA/LAMA + IC). In addition, based on the demand for medical care, non-disease factors may have influenced the results, such as access to health resources, comorbidity or patient specifics, which could cause worsening episodes not to be reported by the patient and therefore remain untreated. We cannot exclude an indication bias due to severity, but when we adjust for parameters related with severity it does not seem to be any relevant difference. Regarding the changes in medication, the modifications of the concomitant respiratory treatment were not considered either, due to the technical complexity (time period) that represented their quantification in the database. Unfortunately, a more detailed analysis by molecules was not possible due to the small sample size. However, this study reflects routine clinical practice, in which the degree of adherence to clinical practice guidelines is rather low. In fact, in clinical practice it is common that more than $50 \%$ of patients are taking the high dose triple in GOLD, which is not in accordance with the (GOLD) guidelines. ${ }^{35}$

In conclusion, triple therapy may be an appropriate option in many patients, with low doses of IC. Increased IC doses were not associated with a reduction in the number of exacerbations, while an increased risk of pneumonia was observed. Patients treated with extrafine particle devices had lower rates of severe and moderate exacerbations, resulting in lower health costs for the Spanish National Health System. These results should be replicated in other healthcare settings.

\section{Abbreviations}

ATC, Anatomical Therapeutic Chemical Classification System; COPD, Chronic obstructive pulmonary disease; $\mathrm{FEV}_{1}$, Maximum expiratory volume in the first second; GOLD, Global Initiative for Obstructive Lung Disease; HCRU, Healthcare resource use; ICS, Inhaled corticosteroid; LABA, Long-acting beta-2 agonist; LAMA, Long-acting muscarinic antagonist; LABD, Long-acting bronchodilators; MMAD, Mass median aerodynamic diameter; SAMA, Shortacting muscarinic antagonist.

\section{Funding}

This study was funded by Chiesi España S.A.U.

\section{Disclosure}

ASM reports grants from Chiesi, outside the submitted work. FJdA reports grants from Sanofi-Pasteur, outside the submitted work. JLIA has received consulting or speaking fees from AstraZeneca, Bayer, Boehringer Ingelheim, Chiesi, GSK, Grifols, Menarini, Novartis, Orion, Pfizer, Sandoz and Teva.The authors report no other conflicts of interest in this work.

\section{References}

1. Vogelmeier CF, Criner GJ, Martinez FJ, et al. Global strategy for the diagnosis, management, and prevention of Chronic Obstructive Lung Disease 2017 report. GOLD executive summary. Am J Respir Crit Care Med. 2017;195:557-582. doi:10.1164/rccm.201701-0218PP

2. Kiley JP, Gibbons GH. COPD national action plan: addressing a public health need together. Chest. 2017;152:698-699. doi:10.1016/j. chest.2017.08.1155

3. Blanco I, Diego I, Bueno P, et al. Geographical distribution of COPD prevalence in Europe, estimated by an inverse distance weighting interpolation technique. Int $J$ Chron Obstruct Pulmon Dis. 2017;13:57-67. doi:10.2147/COPD.S150853

4. Alfageme I, de Lucas P, Ancochea J, et al. 10 years after EPISCAN: a new study on the prevalence of COPD in Spain -a summary of the EPISCAN II protocol. Arch Bronconeumol. 2019;55:38-47. doi:10.1016/j.arbres.2018.05.011 
5. Sicras-Mainar A, Rejas-Gutiérrez J, Navarro-Artieda R, Ibáñez-Nolla J. The effect of quitting smoking on costs and healthcare utilization in patients with chronic obstructive pulmonary disease: a comparison of current smokers versus ex-smokers in routine clinical practice. Lung 2014;192:505-518. doi:10.1007/s00408-014-9592-7

6. Miravitlles M, Murio C, Guerrero T, Gisbert R; on behalf of the DAFNE study group. Costs of chronic bronchitis and COPD. A one year follow-up study. Chest. 2003;123:784-791. doi:10.1378/ chest.123.3.784

7. Sicras A, Huerta A, Navarro R, Ibañez J. Use of resources and associated costs of chronic obstructive pulmonary disease exacerbations: A population based retrospective study. Semergen. 2014;40:189-197. doi:10.1016/j.semerg.2013.10.002

8. Global Initiative for Chronic Obstructive Lung Disease (GOLD). From the global strategy for the diagnosis, management and prevention of COPD; 2019. Available from: http://goldcopd.org. Accessed August 18, 2019.

9. Miravitlles M, Soler-Cataluña JJ, Calle M, et al. Spanish guidelines for management of Chronic Obstructive Pulmonary Disease (GesEPOC) 2017. pharmacological treatment of stable phase. Arch Bronconeumol. 2017;53:324-335. doi:10.1016/j.arbres.2017.03.018

10. Singh D, Papi A, Corradi M, et al. Single inhaler triple therapy versus inhaled corticosteroid plus long-acting $\beta 2$-agonist therapy for chronic obstructive pulmonary disease (TRILOGY): a double-blind, parallel group, randomised controlled trial. Lancet. 2016;388:963-973. doi:10.1016/S0140-6736(16)31354-X

11. Lipson DA, Barnacle H, Birk R, et al. FULFIL trial: once-daily triple therapy in patients with Chronic Obstructive Pulmonary Disease. Am $J$ Respir Crit Care Med. 2017;196:438-446. doi:10.1164/ rccm.201703-04490C

12. Alcázar Navarrete B, Casanova C, Miravitlles M, de Lucas P, Riesco JA, Rodríguez González-Moro JM; Working Group. "Consensus document on the appropriate use of inhaled corticosteroids in COPD". Correct use of inhaled corticosteroids in chronic obstructive pulmonary disease": a consensus document. Arch Bronconeumol. 2015;51:193-198. doi:10.1016/j.arbres.2014.11.001

13. Magnussen H, Disse B, Rodriguez-Roisin R, et al. WISDOM Investigators. Withdrawal of inhaled glucocorticoids and exacerbations of COPD. $N$ Engl J Med. 2014;371:1285-1294. doi:10.1056/ NEJMoa1407154

14. Watz H, Tetzlaff K, Wouters EF, et al. Blood eosinophil count and exacerbations in severe chronic obstructive pulmonary disease after withdrawal of inhaled corticosteroids: a post-hoc analysis of the WISDOM trial. Lancet Respir Med. 2016;4:390-398. doi:10.1016/ S2213-2600(16)00100-4

15. Lipworth B, Manoharan A, Anderson W. Unlocking the quiet zone: the small airway asthma phenotype. Lancet Respir Med. 2014;2:497. doi:10.1016/S2213-2600(14)70103-1

16. Dhand R, Cavanaugh T, Skolnik N. COPD in primary care: key considerations for optimized management: considerations for optimal inhaler device selection in Chronic Obstructive Pulmonary Disease. J Fam Pract. 2018;67(2 Suppl):S19-S27.

17. Singh D, Corradi M, Spinola M, et al. Triple therapy in COPD: new evidence with the extrafine fixed combination of beclomethasone dipropionate, formoterol fumarate, and glycopyrronium bromide. Int J Chron Obstruct Pulmon Dis. 2017;12:2917-2928. doi:10.2147/ COPD.S146822

18. Bassam M, Mayank V. Glucocorticoids - new recognition of our familiar friend. Chapter 23. Steroids in asthma: friend or foe; 2012 Available from: https://www.intechopen.com/books/glucocorticoidsnew-recognition-of-our-familiar-friend/steroids-in-asthma-friend-orfoe. Accessed September 10, 2019.

19. Charlson ME, Pompei P, Ales KL, Mackenzie CR. A new method of classifying prognostic comorbidity in longitudinal studies: development and validation. J Chronic Dis. 1987;40:373-383. doi:10.1016/ 0021-9681(87)90171-8
20. World Health Organization. The anatomical therapeutic chemical classification system with defined daily doses (ATC/DDD): disponible en; 2020. http://www.who.int/classifications/atcddd/en/. Consultado: enero.

21. Vanfleteren L, Fabbri LM, Papi A, Petruzzelli S, Celli B. Triple therapy (ICS/LABA/LAMA) in COPD: time for a reappraisal. Int $J$ Chron Obstruct Pulmon Dis. 2018;13:3971-3981. doi:10.2147/COPD.S185975

22. Cazzola M, Calzetta L, Rogliani P, Matera MG. Triple therapy versus dual bronchodilation and inhaled corticosteroids/long-acting $\beta$-agonists in COPD: accumulating evidence from network meta-analyses. Pulm Ther. 2019;5:117-126. doi:10.1007/s41030-019-00102-8

23. Izquierdo JL, Cosio BG. The dose of inhaled corticosteroids in patients with COPD: when less is better. Int $J$ Chron Obstruct Pulmon Dis. 2018;13:3539-3547. doi:10.2147/COPD.S175047

24. Cheng SL, Su KC, Wang HC, Perng DW, Yang PC. Chronic obstructive pulmonary disease treated with inhaled medium- or high-dose corticosteroids: a prospective and randomized study focusing on clinical efficacy and the risk of pneumonia. Drug Des Devel Ther. 2014;8:601-607. doi:10.2147/DDDT.S63100

25. Morros R, Vedia C, Giner-Soriano M, Casellas A, Amado E, Baena JM; en representación de los investigadores del proyecto PNEUMOCORT. Community-acquired pneumonia in patients with chronic obstructive pulmonary disease treated with inhaled corticosteroids or other bronchodilators. Study PNEUMOCORT. Aten Primaria. 2019;51:333-340. doi:10.1016/j.aprim.2018.02.007

26. Vestbo J, Papi A, Corradi M, et al. Single inhaler extrafine triple therapy versus long-acting muscarinic antagonist therapy for chronic obstructive pulmonary disease (TRINITY): a double-blind, parallel group, randomised controlled trial. Lancet. 2017;389 (10082):1919-1929. doi:10.1016/S0140-6736(17)30188-5

27. Wedzicha JA, Singh D, Vestbo J, et al. FORWARD Investigators. Extrafine beclomethasone/formoterol in severe COPD patients with history of exacerbations. Respir Med. 2014;108:1153-1162. doi:10.1016/j.rmed.2014.05.013

28. Papi A, Vestbo J, Fabbri L, et al. Extrafine inhaled triple therapy versus dual bronchodilator therapy in chronic obstructive pulmonary disease (TRIBUTE): a double-blind, parallel group, randomised controlled trial. Lancet. 2018;391(10125):1076-1084. doi:10.1016/ S0140-6736(18)30206-X

29. Scichilone N, Benfante A, Morandi L, Bellini F, Papi A. Impact of extrafine formulations of inhaled corticosteroids/long-acting beta-2 agonist combinations on patient-related outcomes in asthma and COPD. Patient Relat Outcome Meas. 2014;5:153-162.

30. Press VG, Konetzka RT, White SR. Insights about the economic impact of chronic obstructive pulmonary disease readmissions post implementation of the hospital readmission reduction program. Curr Opin Pulm Med. 2018;24:138-146. doi:10.1097/MCP.0000000000000454

31. Ehteshami-Afshar S, FitzGerald JM, Doyle-Waters MM, Sadatsafavi M. The global economic burden of asthma and chronic obstructive pulmonary disease. Int J Tuberc Lung Dis. 2016;20 (1):11-23. doi:10.5588/ijtld.15.0472

32. Sicras Mainar A, Huerta A, Navarro Artieda R, Monsó E, Landis SH, Ismaila AS. Economic impact of delaying initiation with multiple-inhaler maintenance triple therapy in Spanish patients with chronic obstructive pulmonary disease. Int J Chron Obstruct Pulmon Dis. 2019;14:2121-2129. doi:10.2147/COPD.S211854

33. Iheanacho I, Zhang S, King D, Rizzo M, Ismaila AS. Economic burden of Chronic Obstructive Pulmonary Disease (COPD): a systematic literature review. Int J Chron Obstruct Pulmon Dis. 2020;15:439-460. doi:10.2147/COPD.S234942

34. Trudo F, Kallenbach L, Vasey J, et al. Clinical and economic burden of eosinophilic COPD in a large retrospective US cohort. Int J Chron Obstruct Pulmon Dis. 2019;14:2625-2637. doi:10.2147/COPD.S220009

35. Izquierdo JL, Morena D, González Y, et al. Clinical management of COPD in a real-world setting. A big data analysis. Arch Bronconeumol. 2020:S0300-2896(20)30012-0. doi:10.1016/j. arbres.2019.12.025. 


\section{Publish your work in this journal}

The International Journal of COPD is an international, peer-reviewed journal of therapeutics and pharmacology focusing on concise rapid reporting of clinical studies and reviews in COPD. Special focus is given to the pathophysiological processes underlying the disease, intervention programs, patient focused education, and self management protocols. This journal is indexed on PubMed Central, MedLine and CAS. The manuscript management system is completely online and includes a very quick and fair peer-review system, which is all easy to use. Visit http://www.dovepress.com/testimonials.php to read real quotes from published authors. 